\title{
The access of relevant information for solving problems
}

\author{
BRIAN H. ROSS, WILLIAM J. RYAN, and PATRICIA L. TENPENNY \\ University of Illinois, Champaign, Illinois
}

\begin{abstract}
Two issues were investigated concerning the retrieval of recently acquired relevant information for solving riddle problems. First, although earlier research indicated that problem solvers often fail to retrieve relevant information unless they are explicitly informed of its relevance, Bowden (1985) suggested that uninformed subjects would benefit greatly from additional problemsolving time. In two experiments, we found that uninformed subjects solved more problems than did subjects who did not receive the information, but a simple model attributed this difference to these uninformed subjects "catching on" to the information's relevance after solving some problems. Second, the retrieval characteristics were examined by varying the proportion of problems for which clues were given. Informed subjects given clues for only half of the problems benefited (though perhaps not fully) from being informed, without incurring any cost from being misdirected on the unclued problems. The discussion focuses on some ways in which accessing relevant information may affect problem solving.
\end{abstract}

How people access and use relevant knowledge is a central issue in cognition. As the emphasis in problemsolving research has shifted to the knowledge required to solve problems, increasing attention has been focused on questions about the access and use of such knowledge (e.g., Adams et al., 1988; Gentner \& Landers, 1985; Gick \& Holyoak, 1980, 1983; Holyoak \& Koh, 1987; Lockhart, Lamon, \& Gick, 1988; Perfetto, Bransford, \& Franks, 1983; Ross, 1984, 1987, 1989a, 1989b; Stein, Way, Benningfield, \& Hedgecough, 1986; Weisberg, DeCamillo, \& Phillips, 1978). A number of these studies have shown that subjects often fail to transfer relevant knowledge spontaneously to a new situation. Although this lack of transfer could be due either to failure to access the relevant information or to failure to use it once accessed, in many cases people fail to access the relevant information (though see Ross, 1987, 1989a, and Stein et al., 1986, for difficulties with the use of accessed information). These failures of access occur when the earlier information is analogous to the current problem (e.g., Gick \& Holyoak, 1980, 1983; Reed, Ernst, \& Banerji, 1974; Spencer \& Weisberg, 1986) as well as when the earlier information is not analogous, but is very relevant to the current problem (e.g., Perfetto et al., 1983).

It may be useful to make this idea more concrete with an example that is closely related to the current investigation. Perfetto et al. (1983) provided evidence that sub-

This research was supported by NSF Grant IST-8308670. We thank Doug Medin, Ed Shoben, Gordon Logan, and Dedre Gentner for discussions about this research. We also thank Robert Weisberg, Edward Bowden, and an anonymous reviewer for their helpful reviews, and Marna Smith for help in conducting and analyzing the experiments. Address correspondence and requests for reprints to Brian H. Ross, Department of Psychology, University of Ilinois, 603 E. Daniel, Champaign, IL 61820 . jects who are uninformed of the relevance of learned information often fail to access that information spontaneously during problem solving. Their results were surprising in that the earlier information seemed very relevant to the problem-solving task and preceded it by only a few minutes. In those studies, subjects first rated the truthfulness of 14 sentences such as, "A minister marries several people each week." A few minutes later, the subjects were asked to solve 15 riddles such as, "A man who lived in a small town in the U.S. married 20 different women of the same town. All are still living and he has never divorced one of them. Yet, he has broken no law. Can you explain?' The subjects in the experiment who were not informed of the relevance of the earlier sentences solved far fewer riddles than did those subjects who were informed. Moreover, these uninformed subjects did not solve reliably more riddles than did subjects who did not see the earlier sentences. Thus, in the Perfetto et al. studies, explicitly informing subjects of the relevance of the earlier sentences was a necessary condition for obtaining transfer.

Information learned in one context must often be spontaneously accessed in another context. Research concerned with the failure to access relevant information has proceeded along two related lines. The first line has tried to identify the factors that might improve access, such as the way in which the relevant information is processed (e.g., Adams et al., 1988; Lockhart et al., 1988), surface similarity (e.g., Gentner \& Landers, 1985; Holyoak \& Koh, 1987; Ross, 1984, 1987; Stein et al., 1986), context (Spencer \& Weisberg, 1986), and the comparison of instances (Dellarosa, 1985; Gick \& Holyoak, 1983). The second related line of research has probed the nature of the retrieval process in these problem-solving situations. Although this approach overlaps considerably with the 
first approach, especially in the examination of surface similarities and context, it also considers issues such as interference during the retrieval of relevant information (e.g., Ross, 1984, Experiment 3) and the importance of the relative similarity between the problem and relevant information (Ross, 1987, Experiment 2). Because manipulating whether subjects are informed of the relevance of the earlier sentences affects access, this paradigm provides an opportunity to explore the retrieval process in more detail without varying the relevant information provided.

Recently, Bowden (1985) proposed that the diffiulty of spontaneously accessing relevant information might not be as great as these other studies suggest. He suggested that the time constraints of some of these studies might have led to underestimating access for the uninformed subjects. Bowden argued that the informed subjects had a very constrained set of information to search, so that they were likely to access the relevant information more quickly than were the uninformed subjects. However, according to Bowden, these uninformed subjects, given more time, should succeed in accessing the relevant information as well. Thus, the difference between these groups was not access versus nonaccess of relevant information, but faster versus slower access of relevant information. Bowden provided evidence for this assertion by showing, with materials and procedure similar to those employed by Perfetto et al. (1983), that when subjects were given more time to solve the riddles, the informed-uninformed difference disappeared. The timing data provided further support for his contention. If the subjects had been stopped very early in their problem solving, the pattern of Perfetto et al. would have been obtained (i.e., uninformed performance would be worse than informed, but would not be reliably better than baseline subjects who did not get the earlier sentences).

Bowden's (1985) findings are interesting for two reasons. First, they call into question the conclusion of previous investigators who found that uninformed subjects will not usually access relevant information spontaneously. It is important to realize that these earlier studies did not show that people never spontaneously access relevant information, but that in cases in which it seems as if they will access such information, they often do not. These demonstrations indicate that we may be seriously underestimating the difficulty of accessing relevant information. Bowden's findings suggest that failure to access may not be so fundamental and may be rectified by increasing the time that uninformed subjects have for problem solving. Proponents of the access-difficulty view might object to the generality of Bowden's findings on the grounds that his clues were blatant (even more so than those of Perfetto et al., 1983) and had been seen very recently. In addition, people do not spend unlimited time trying to solve problems, so even if the problem-solving difference is largely due to the time to access the relevant information, there may be important consequences in many problem-solving situations.

A second interesting aspect of Bowden's (1985) paper is the proposal that the explicit cluing of the earlier sen- tences served to direct the search of the informed subjects. The search space of uninformed subjects was not restricted, so these subjects should have taken longer to find the relevant information. Unlike many accounts, this proposal explains why providing additional time allowed the performance of the uninformed subjects to equal (or almost equal) the performance of the informed subjects. Bowden noted that this result would also be expected if uninformed subjects simply "caught on" to the relevance of the earlier sentences, but he provided some evidence (to be discussed after Experiment 1) against this alternative account. Note, however, that if the uninformed subjects were not catching on, it is puzzling that the informed and the uninformed subjects performed equally well when only the former group realized that the earlier sentences were relevant. This point will be dealt with in more detail before Experiment 2. Much of the other research on the access of relevant information has been concerned with the conditions and manipulations affecting transfer, not with examining the retrieval process and its properties (though see Weisberg et al., 1978). These retrieval properties will be addressed again before Experiment 3 .

\section{EXPERIMENT 1}

Experiment 1 initiated our study of the retrieval properties of accessing relevant information by replicating the conditions of Bowden's (1985) study.

\section{Method}

Design. Two groups of subjects rated sentences (which included clues to the 10 critical riddles) for truthfulness. Following a short intervening task, the subjects attempted to solve 13 riddles ( 3 "buffers" and 10 "critical"), with a time limit of 2 min per riddle. One group of subject was informed of the relevance of the earlier sentences and the other group was uninformed. A third group of baseline subjects simply solved the riddles without having seen the relevant sentences. The dependent measure of interest is the proportion of congruent solutions (i.e., solutions suggested by the sentences) to the 10 critical riddles. This measure was obtained every $20 \mathrm{sec}$ for $2 \mathrm{~min}$.

Materials. The riddles were the 10 used by Bowden (1985) and listed in the Appendix of his article (these riddles were originally taken from Gardner, 1978). The 10 sentences that clued the riddles were also the ones used by Bowden. Three additional riddles were taken from Rosenbloom (1976) to be the first three buffer riddles presented, as in Bowden's (1985) study. Five additional sentences were constructed as fillers for the sentence-rating task.

The sentence-rating materials were five pages with three sentences on each page. The riddles were typed one to a page. The booklet of riddles contained the 3 filler riddles, followed by the 10 critical riddles in exactly the same order as in Bowden's (1985) study to provide as close a replication as possible. ${ }^{1}$ The front sheets of both the sentence-rating and the riddle booklets asked for the date and the subject's name.

Procedure. The subjects were tested in groups of 6 to 12, using Bowden's (1985) procedure. The subjects in the informed and the uninformed groups first rated the truthfulness of the 15 sentences on a 5-point scale. They were allowed $20 \mathrm{sec}$ to rate each sentence. Following this task, they spent $5 \mathrm{~min}$ on an unrelated (letter-series completion) task.

The subjects in all groups were then given the riddle booklets. They spent 2 min on each riddle and were not allowed to go on to the next riddle until the $2 \mathrm{~min}$ had elapsed. The subjects were 
instructed to write any solution they thought was correct. As in Bowden's (1985) study, to estimate the time when each solution was written, the subjects were asked to draw a line across the page below all of their solutions every $20 \mathrm{sec}$, which was signaled by the experimenter. They were told to write all answers below the last line they had drawn. The informed group was then told that some of the sentences they had rated earlier might be useful in solving these riddles.

A brief questionnaire was filled out following the riddle task. The subjects were asked if they had seen any of the riddles before the experiment. In addition, the uninformed subjects were asked if they had noticed the relationship between the sentences and the riddles and, if so, on which riddle this relationship was first noticed.

Subjects. The subjects were 120 University of Illinois undergraduates who participated as part of a course requirement, 40 subjects in each group. ${ }^{2}$ The data from 4 additional subjects ( 2 from the uninformed group and 1 each from the other groups) who had prior knowledge of more than two of the critical riddles were not used.

\section{Results and Discussion}

The purpose of this experiment was to replicate Bowden's (1985) results. The 10 critical riddles were scored for congruent solutions and the time interval at which the congruent solution occurred was recorded. Figure 1 presents these data for the three groups in cumulative curves over the six 20 -sec intervals. A significance level of .05 is used throughout.

The first analysis was conducted on the proportions of congruent solutions at the end of the 2-min interval. These proportions were $.798(S D=.13), .645(S D=$ $.19)$, and $.518(S D=.23)$ for the informed, uninformed, and baseline groups, respectively $[F(2,117)=21.66$, $\left.M S_{\mathrm{e}}=.037\right]$. Contrary to the results of Bowden (1985), but consistent with Perfetto et al.'s (1983) findings, the informed group solved reliably more riddles than did the uninformed group $[t(78)=4.11] .{ }^{3}$ However, consistent with Bowden's finding and contrary to that of Perfetto et al., the uninformed group's performance exceeded that of the baseline group $[t(78)=2.66]$.

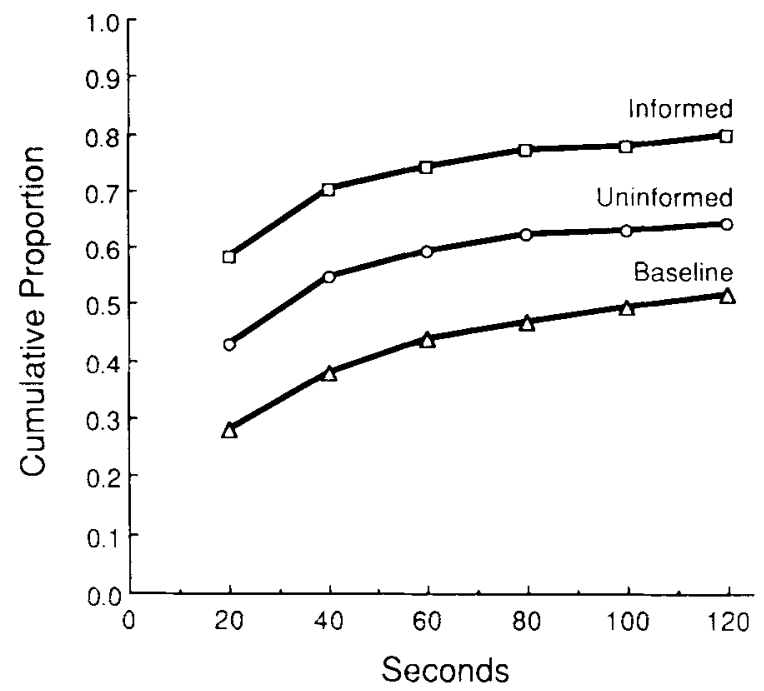

Figure 1. Cumulative proportions of congruent solutions in $\mathbf{E x}$ periment 1 at 20-sec intervals.
Table 1

Percentage of Congruent Solutions and Mean Time Interval of Solutions in Experiment 1 for Each Solved Riddle

\begin{tabular}{|c|c|c|c|c|c|c|}
\hline \multirow[b]{3}{*}{ Riddle } & \multicolumn{6}{|c|}{ Group } \\
\hline & \multicolumn{2}{|c|}{ Informed } & \multicolumn{2}{|c|}{ Uninformed } & \multicolumn{2}{|c|}{ Baseline } \\
\hline & Percentage & Time & Percentage & Time & Percentage & Time \\
\hline 1 & 98 & 1.44 & 88 & 1.29 & 70 & 1.96 \\
\hline 2 & 83 & 1.36 & 85 & 1.56 & 55 & 1.64 \\
\hline 3 & 98 & 1.26 & 95 & 1.13 & 93 & 1.38 \\
\hline 4 & 45 & 2.00 & 43 & 1.82 & 43 & 1.59 \\
\hline 5 & 88 & 1.60 & 43 & 2.12 & 25 & 4.20 \\
\hline 6 & 60 & 1.75 & 30 & 1.58 & 13 & 3.20 \\
\hline 7 & 78 & 1.19 & 58 & 1.57 & 48 & 2.32 \\
\hline 8 & 100 & 1.28 & 83 & 1.46 & 70 & 1.96 \\
\hline 9 & 90 & 1.50 & 65 & 1.73 & 40 & 1.81 \\
\hline 10 & 65 & 2.46 & 58 & 2.83 & 65 & 2.65 \\
\hline
\end{tabular}

Note $-n=40$ /group; Percentage $=$ percent of congruent solutions; Time $=$ mean solution-time interval, where Interval 1 is $0-20 \mathrm{sec}$, Interval 2 is $21-40 \mathrm{sec}$, up to Interval 6 for riddles solved in 101-120 sec. Riddles that were not solved are not included in the time calculations, although their effect is discussed in the text.

The cumulative curves of Figure 1 are also inconsistent with the timing data of Bowden (1985). He found that, initially, the performance of the uninformed group was similar to that of the baseline group, but that, by the end of the 2-min period, the performance of the uninformed group was similar to that of the informed group. In the present study, the curves are nearly parallel, but that of the baseline group increases slightly faster than those of the other two groups after the first two intervals. The informed group produced more congruent solutions at every interval than did the uninformed group $[t \mathrm{~s}(78)>3.22]$, and this advantage did not decrease with time. The uninformed group had a higher proportion of congruent solutions than did the baseline group at every interval $[t \mathrm{~s}(78)>2.66]$, but the differences did not increase with time. ${ }^{4}$ Thus, although the timing data of Bowden's study were not replicated, there was an uninformed-baseline difference. The exact proportions correct for each interval are provided in Appendix A.

Following Bowden (1985), we further examined the group differences. Table 1 shows the proportion of congruent solutions and mean solution times for each riddle. Mean solution times for solved riddles were calculated by assigning numbers to the intervals (i.e., 1 for first $20 \mathrm{sec}, 2$ for next $20 \mathrm{sec}$, etc., up to 6 for solution in last $20 \mathrm{sec}) .{ }^{5}$ As can be seen, the informed group produced more congruent solutions than did the uninformed group on 9 of the 10 riddles, and the uninformed group performed better than the baseline group on 8 of 9 riddles with one tie. The informed group times were faster than those of the uninformed group on only 6 of 10 riddles, whereas the uninformed group times were faster than those of the baseline group on 8 of 10 riddles (if a 7 is assigned for nonsolutions, these solution-time differences are 9 of 10 and 8 of 10 , respectively). The riddle by riddle analysis was generally consistent with the overall analyses, with the informed group showing the highest per- 
formance and the uninformed group performing marginally higher than the baseline group.

The questionnaire indicated that in the uninformed group, 29 of 40 subjects had caught on that the earlier sentences could be used to help solve the riddles. These data will be discussed shortly, but it should be noted that the proportion (.725) is similar to that reported by Bowden (1985) $(.70)$.

In summary, the informed group produced more congruent solutions to the riddles than did the uninformed group, contrary to the findings of Bowden (1985). Moreover, the uninformed group performed reliably better than the baseline group, contrary to Perfetto et al.'s (1983) findings and consistent with Bowden's data. (Perfetto et al.'s findings indicate a nonreliable difference in this direction, but Bowden's cues were more blatantly relevant than were Perfetto et al.'s.) Finally, the present experiment provided no evidence for any major change in the differences over time as Bowden found. (All of these findings will be replicated in Experiment 2.) The reasons for these differences from Bowden (1985) are not clear, although his experiment did have less power than did the current studies (only half as many subjects).

Much of the research mentioned earlier has focused on the conditions under which transfer may occur, so a case in which uninformed subjects outperform baseline subjects is important if we can understand the source of this difference. Bowden (1985) suggested that more time might be sufficient to produce this difference, but in our study, additional time was not necessary: the uninformed subjects performed better even in the early intervals. An important aspect of the condition in which spontaneous transfer does not occur is that the subjects not be aware of the relevance of the earlier information. Thus, when transfer does occur, it is necessary for the researcher to demonstrate that the subjects did not become aware of the relevance (i.e., catch on). Bowden reasoned that if uninformed subjects caught on during the experiment, their performance would change more than would that of the other two groups from the first five riddles to the second five riddles. He found no evidence of this greater change, and so argued against the idea that his results were caused by such catching on. Although 14 of his 20 uninformed subjects did claim to have caught on, he reasonably noted that these reports may have been inaccurate. In what follows, we show that the idea that uninformed subjects do catch on is sufficient to account for much of his data. We then use a particular form of this suggestion to account for the results of the first experiment and to motivate the next experiment.

First, we examined whether the subjects in Bowden's (1985) uninformed condition might have performed the way they did because they caught on. To assess this possibility, we used a simple mixture model. We assumed that if the uninformed subjects did not catch on, they were exactly like subjects in the baseline condition, and if they did catch on, they were exactly like subjects in the informed condition. We used the subject reports to estimate the probability of catching on $(14 / 20=.7)$. This simple mixture model (i.e., predicting the uninformed group performance as a weighted average of that of the baseline and the informed groups, with weights of .3 and .7, respectively) predicts an overall proportion of .514 (observed, .54) and a mean interval to solution of 4.26 , using an unweighted mean of correct solutions (observed, 4.40). The model cannot account for the changes with time intervals that Bowden observed (since the mixture is constant), but our Experiment 1 did not replicate these changes.

In an effort to understand these effects, we tested a few other uninformed subjects who were asked to think aloud as they tried to solve the riddles. Some of these subjects appeared to catch on to the relevance of the clues, but only after solving a riddle. That is, they apparently noticed the similarity between their answer and an earlier sentence they had rated. In addition, this noticing usually occurred in the first three critical (nonbuffer) riddles. As can be seen in Table 1, as well as in Bowden's (1985) article, the first three riddles were relatively easy, even for the baseline group. We suggest that the uninformed subjects may have solved one or more of these early riddles without the use of the earlier sentences, and that the answers to these riddles may have cued some of the uninformed subjects to the relevance of the clues. (A similar suggestion has recently been made by Lockhart et al., 1988, to account for some of their data.)

This hypothesis is a modified mixture model, in which all uninformed subjects begin as baseline subjects. If we knew when the subjects in the uninformed condition caught on, we would predict that their performance on the later riddles would be as in the informed group. Thus, the overall performance of the uninformed group would again be a mixture of the other two groups, but the probabilities would not simply be the proportion of subjects who did and did not catch on, but the proportion of riddles for which the subjects had or had not already caught on. For example, if a subject caught on at the third riddle, s/he would be assumed to be a mixture of baseline (for the first three riddles) and informed (for the last seven riddles).

Therefore, to ascertain when the subjects caught on, we used a postexperiment questionnaire that asked the subjects to identify the riddle at which they had caught on (if they had caught on at all). Of the 40 uninformed subjects, 29 claimed to have caught on. Although it is impossible to know for certain how accurate these selfreports are, it is encouraging that 28 of these 29 subjects identified 1 of the 10 critical riddles rather than one of the three buffers for which no clues were given.

To save space, only the main results of the model fits will be described here; more detailed information is provided in Appendix A. ${ }^{6}$ The predicted prope... ion of final solutions for the uninformed group was .678 , which is close to the observed proportion of .645. As shown in Appendix A, the predictions for the five earlier intervals were also very close, with differences ranging from .016 to .032. Both $\chi^{2}$ and $r^{2}$ (see Appendix A) were computed 
as goodness-of-fit indexes, and both indicated that the model did a good job of predicting the data $\left[\chi^{2}(6)=6.83\right.$, $p>.3 ; r^{2}=.988$ ]. Finally, in line with our suggestion that Bowden's (1985) subjects may have caught on because the first three riddles were fairly easy, 24 of the 28 subjects in our experiment who reported catching on at a critical riddle identified one of the first three. Fifteen of these subjects said they had caught on at the very first critical riddle. Taken together, these results cast doubt on the possibility that the uninformed-baseline difference is due to the subjects' accessing the clue sentences without becoming aware of their relevance. Despite this evidence for catching on, the breakdown by riddle halves as done by Bowden shows no greater change for the uninformed group relative to the other groups (see Appendix B for details).

\section{EXPERIMENT 2}

Experiment 2 was performed to provide a second attempt to replicate Bowden's (1985) results and to test the mixture model's explanation of performance for the uninformed group of subjects. As noted earlier, it seemed that the first three riddles might be particularly effective in getting uninformed subjects to notice the relevance of the earlier sentences. If so, then moving these riddles to the end of the list should lead subjects to catch on later and therefore should reduce the uninformed-baseline difference. Because the uninformed group's performance was still expected to be a mixture of the informed and the baseline groups' performance (though with more weight on the latter than in Experiment 1), it was not possible to predict that the uninformed-baseline difference would disappear.

\section{Method}

Design, Materials, and Procedure. The design, materials, and procedure were exactly as in Experiment 1, except that the first three critical riddles were moved to the last three positions. ${ }^{7}$

Subjects. The subjects were 123 University of Illinois undergraduates who participated as part of a course requirement and were tested in groups of 5 to 12 . There were 41 subjects in each of the three conditions

\section{Results and Discussion}

The scoring and analyses were as in Experiment 1 . The cumulative curves and riddle data are presented in Figure 2 and Table 2. Again, there was a strong advantage for the informed group over the uninformed group, and some advantage for the uninformed group over the baseline group. ${ }^{8}$

The mean proportions of congruent solutions for the final interval were $.734(S D=.15), .578(S D=.25)$, and $.481(S D=.20)$ for the informed, uninformed, and baseline groups, respectively $[F(2,120)=67.12$, $\left.M S_{\mathrm{e}}=.042\right]$. By pairwise comparisons, the informeduninformed difference was reliable $[t(80)=3.45]$ and the uninformed-baseline difference just missed statistical significance $[t(80)=1.95, p<.06]$.

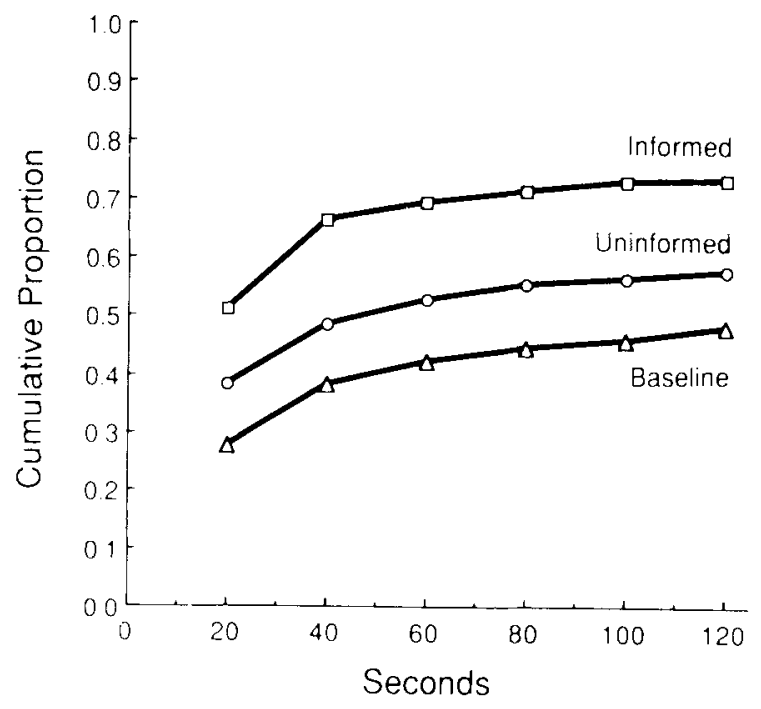

Figure 2. Cumulative proportions of congruent solutions in Experiment 2 at 20 -sec intervals.

The cumulative curves in Figure 2 show that the differences existed throughout (for $20 \mathrm{sec}$ to $100 \mathrm{sec}$, all pairwise $t \mathrm{~s}>2.10$ ); there was no evidence of a change over time. The riddle data in Table 2 show that the informeduninformed difference held for all 10 of the riddles in the proportion-correct data, but for only 7 of 9 (with 1 tie) in the solution-time data. The uninformed group's performance was better than the baseline group's on 7 of 10 riddles, and their solution times were faster on 9 of 10 riddles. (If nonsolutions are included in a seventh interval, the solution times show 10 of 10 and 8 of 10 advantages, respectively.)

The questionnaires indicated that 30 of the 41 subjects in the uninformed group had caught on to the sentences being riddle clues. However, unlike in the earlier experiment, the riddle at which they caught on was scattered throughout the list $(2,3,3,5,2,3,0,4,3,3$ for riddles in Positions 1 through 10, respectively, with 2 subjects indicating one of the buffer riddles for which there were no clues) and 10 of 30 claimed to have caught on during the last three riddles (which had been the first three riddles in Experiment 1). The mixture model does an excellent job of fitting these data $\left[\chi^{2}(6)=3.34, p>.7\right.$; $r^{2}=.995$. (See Appendix A for details.) The prediction for the final interval (.571) was only .007 less than the observed .578. Across intervals, the predicted values were slightly less than the observed values; the differences ranged from .002 to .022 , with a mean of less than .010 . Again, the analysis by riddle halves was not sensitive to catching on (see Appendix B).

One final analysis was conducted to test the idea that the uninformed subjects' reports of catching on reflected a change in their responses. ${ }^{9}$ Using their reports, we examined their solution times (with a 7 assigned for nonsolutions) for riddles before they claimed to have caught on and for riddles after they claimed to have caught on. (Only the subjects who claimed to have caught on were 
Table 2

Percentage of Congruent Solutions and Mean Time Interval of Solutions in Experiment 2 for Each Solved Riddle

\begin{tabular}{|c|c|c|c|c|c|c|c|}
\hline \multirow[b]{3}{*}{ Position } & \multirow[b]{3}{*}{ Riddle } & \multicolumn{6}{|c|}{ Group } \\
\hline & & \multicolumn{2}{|c|}{ Informed } & \multicolumn{2}{|c|}{ Uninformed } & \multicolumn{2}{|c|}{ Baseline } \\
\hline & & Percentage & Time & Percentage & Time & Percentage & Time \\
\hline 1 & 4 & 37 & 1.80 & 24 & 1.30 & 32 & 1.77 \\
\hline 2 & 5 & 46 & 1.84 & 32 & 2.23 & 15 & 2.33 \\
\hline 3 & 6 & 32 & 2.31 & 20 & 2.63 & 10 & 4.00 \\
\hline 4 & 7 & 76 & 2.03 & 59 & 2.25 & 46 & 2.42 \\
\hline 5 & 8 & 88 & 1.47 & 66 & 2.07 & 68 & 1.46 \\
\hline 6 & 9 & 76 & 1.71 & 46 & 1.37 & 41 & 1.53 \\
\hline 7 & 10 & 90 & 1.49 & 63 & 2.08 & 54 & 2.36 \\
\hline 8 & 1 & 100 & 1.20 & 85 & 1.20 & 59 & 2.17 \\
\hline 9 & 2 & 90 & 1.16 & 88 & 1.56 & 59 & 1.79 \\
\hline 10 & 3 & 100 & 1.02 & 95 & 1.05 & 98 & 1.33 \\
\hline
\end{tabular}

Note $-n=41 /$ group; Riddle numbers refer to earlier experiments and the riddles are given in the Appendix of Bowden (1985); Percentage = percent of congruent solutions; Time $=$ mean solution-time interval, where Interval 1 is $0-20 \mathrm{sec}$, Interval 2 is $21-40 \mathrm{sec}$, up to Interval 6 for riddles solved in 101-120 sec. Riddles that were not solved are not included in the time calculations, although their effect is discussed in the text.

included, and the riddles at which they caught on were not included in either group.) By our analysis of the situation, we would expect the mean time to solution to be longer for the riddles solved before catching on than for the riddles solved after catching on (because the baseline mean solution time was longer than the informed mean solution time). The corresponding means were 4.79 $(S D=2.68)$ and $2.64(S D=2.40)$, for $n s$ of 126 and 146 , respectively. No analyses were conducted because of the varied weighting that the subjects contributed to each mean, but it is clear that the means are in the direction predicted. (It should be noted that because the easier riddles were toward the end in Experiment 2, the "after" group may have done better simply because they contained more of the easier riddles. However, even after these means are corrected by using the baseline group's means, the difference is over .77.) This analysis, then, provides additional support for the idea that the subjects were catching on in the uninformed group and that their reports were reasonable estimates of when they caught on. (This analysis could not be conducted for Experiment 1 because so many subjects caught on during the first few riddies that there were not many observations before catching on.)

Note that a number of findings are consistent with the idea that the first three riddles in Experiment 1 were particularly effective in informing the uninformed subjects about the relevance of the earlier sentences. First, the riddle at which subjects claimed to have caught on changed considerably between Experiments 1 and 2, with subjects in the latter study identifying later riddles. Because of this, the weights for the mixture model shifted heavily toward baseline, with weights of .428 and .644 for the baseline in Experiments 1 and 2, respectively. Second, the difference between the uninformed and the baseline groups decreased from .127 in Experiment 1 to .097 in Experiment 2 , whereas the informed-uninformed difference re- mained constant (.153 and .156). Although the former decrease is not reliable given the variability, it does represent a considerable portion of the difference. Third, for the first seven riddles, the baseline group performed only .063 worse than the uninformed group; that is, the uninformed-baseline difference was largely due to two of the last three riddles (riddles in Positions 8 and 9, with the riddle in Position 10 leading to near ceiling performance in both groups), which were the first three riddles in Experiment 1.

Taken together, the results of Experiments 1 and 2 cast doubt on the idea that uninformed subjects produce as many congruent solutions as do informed subjects, but do so more slowly. First, the informed subjects showed a reliable advantage over the uninformed subjects in both experiments. Second, the interval-timing data showed no shift of uninformed group performance from baseline to informed performance over time. Third, although the uninformed group performed better than the baseline group, a simple mixture model based on the subjects' reports provides close fits to the results and suggests an explanation in which the uninformed subjects caught on to the relevance of the earlier sentences. This last finding provides further support for earlier studies (e.g., Perfetto et al., 1983; Weisberg et al., 1978) that indicated the difficulty subjects had with spontaneously accessing relevant information. If we are to learn what conditions promote access (e.g., Adams et al., 1988; Lockhart et al., 1988), it is also important to understand the conditions under which spontaneous access does not occur.

Before proceeding to the final experiment, it is worth noting again that each of these first two studies used only one order of riddles. Although randomizing the riddle order would increase the generality of the results, these studies were initially conducted to replicate and explain the findings of Bowden (1985), which contradicted much 
earlier research. Therefore, we decided it was more important to replicate his procedure as closely as possible than it was to randomize the riddle order.

\section{EXPERIMENT 3}

Research on the noticing of relevant information for problem solving can be viewed from a memory perspective as well as from a problem-solving one. Researchers examining the noticing of analogous information have suggested means of incorporating noticing into standard memory processes (e.g., Anderson \& Thompson, 1989; Holyoak \& Thagard, 1989). Some researchers investigating the retrieval of relevant, but not analogous, information have also made preliminary suggestions concerning retrieval. Weisberg and Alba (1981), for example, have proposed that a problem acts as a cue for relevant information. As mentioned earlier, Bowden (1985) has outlined a problem space-search view in which information about relevance serves to focus the search. Perfetto et al. (1983) noted that subjects in their informed groups often claimed that the answers to the riddles "popped into" their head without any conscious attempt at searching the earlier sentences.

The original intention of our foray into this research was to examine memory retrieval in uninformed subjects. However, the experiments reported so far suggest that in this paradigm, uninformed retrieval of earlier sentences may be rare unless subjects catch on to their relevance. Thus, it appears that to avoid dealing with some unknown mixture of informed and uninformed cases, retrieval issues need to be studied with informed subjects (as they are with most memory paradigms). As the work on analogies has begun to provide us with an understanding of how abstract structures may affect the access and use of earlier examples, an examination of the relevant, but not analogous, information may provide us with some understanding of how knowledge of the information's relevance may affect its retrieval. A number of manipulations such as the number of sentences read (similar to the fanning effects for interference), the usefulness of the clues, and the blatancy of the relevance of the clues could yield interesting results. However, a particularly interesting, but rarely examined, situation is one in which the problem solver has probabilistic information about the relevance of the earlier sentences. That is, we may often be aware that some knowledge we have is possibly, but not definitely, relevant. How does this knowledge about relevance affect the likelihood (and timing) of solving the problem? The following experiment was motivated by this question. To understand the logic, it is easiest to think of the question as having two parts.

First, if the clued earlier knowledge is relevant to the problem, how do probabilistically clued subjects perform relative to informed subjects? Assuming that performance on the clued riddles is higher than performance on the unclued riddles (which it will be), then these partially clued subjects might perform as well (full benefit) or not as well (some benefit) as the informed subjects. Second, when the clued knowledge is not relevant to the problem, how do these " misdirected" subjects perform relative to the baseline subjects? Is there a cost to this misdirection (performance worse than baseline) or not (performance equal to baseline)? These benefits (some or full) and costs (some or none) could occur in any combination. Their implications for the memory-retrieval processes will be more fully discussed after the results have been presented. For now, let us note that the findings can serve to constrain explanations of the retrieval. For example, if the misdirected subjects showed no cost, a process in which they always searched the possibly relevant set first would have difficulties in accounting for the results. In addition, the combination of costs and benefits can be diagnostic. For instance, the suggestion that the clues "pop out" might indicate full benefit, with little if any cost, whereas Bowden's (1985) suggestion of directed search might predict a tradeoff (i.e., if subjects get full benefit, then they are using the relevance fully, so they should incur some cost; if subjects show no cost, then they are not using the relevance, so they should gain little if any benefit).

\section{Method}

Design. The experiment included three groups of subjects. The baseline and informed groups were as in the other experiments. An additional group was also informed of the relationship between the sentence-rating and the riddle tasks, but only half of the 10 critical riddles had clues in the sentence-rating task. Thus, this group had half of the critical riddles clued and half unclued. This group will be referred to as the misdirected group (though this name is really only true for the unclued riddles). For each group, two different orders of riddles were used. For the misdirected group, crossed with this order manipulation, half of the subject were given clues to one half of the riddles, and the other half of the subjects were given clues to the other half of the riddles.

Materials. The 13 riddles from the first two experiments were used but, because the three critical riddles that were first in Bowden's (1985) study and in Experiment 1 led to such high proportions correct, they were exchanged with the buffer riddles. These new critical riddles are given in Appendix $\mathrm{C}$, along with their clues. The 13 riddles were put in a booklet, with one riddle per page. Following the three new buffer riddles, the 10 critical riddles were ar ranged in one of two orders.

The sentence-rating booklets were made as in the other experiments, but the clues for the new critical riddles were added. For the misdirected group, two different booklets were constructed, each with clues to only half of the critical riddles. The clues for the new buffer riddles were included in all booklets. The remaining 7 of the 15 sentences were generated with the same form as the critical and buffer clues.

Procedure. The procedure was exactly as in the other studies, with the misdirected group treated as an informed group (except for the sentence-rating booklets used). As in the other studies, the subjects in the informed group were told that the earlier sentences would be helpful in solving some of the riddles.

Subjects. The subjects were 104 University of Illinois students who participated as part of a course requirement. They were tested in groups of 9 to 17 . There were 26 subjects in the informed and baseline groups ( 13 with each riddle order) and 52 subjects in the misdirected group (13 in each combination of riddle order $\times$ riddle half clued). 


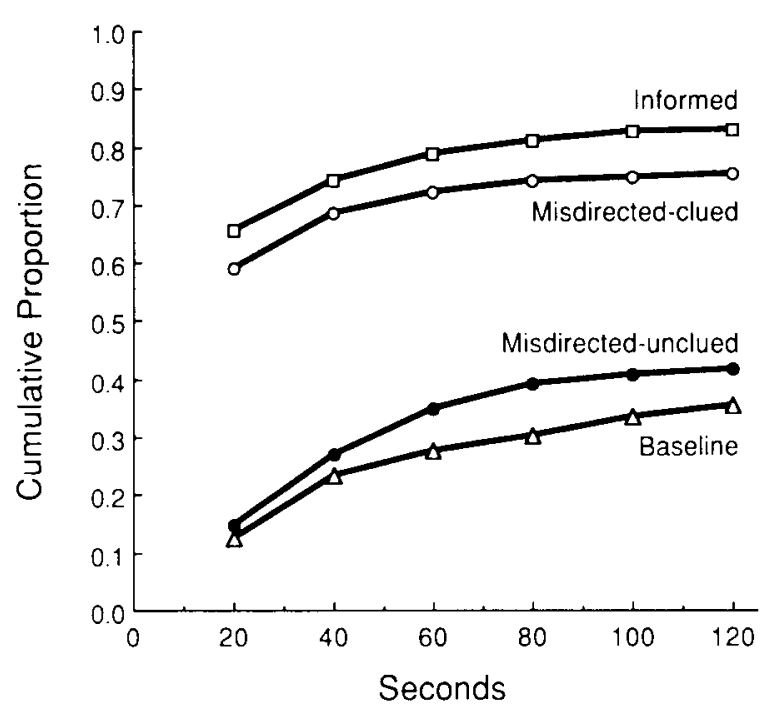

Figure 3. Cumulative proportions of congruent solutions in Experiment 3 at 20 -sec intervals.

\section{Results and Discussion}

The 10 critical riddles were scored for congruent solutions as in the other experiments, and the time intervals of these solutions were recorded. The comparisons of interest are performance by the informed group relative to the clued riddles in the misdirected group and performance by the baseline group relative to the unclued riddles in the misdirected group. To simplify the analyses, the data from 2 subjects in the misdirected group (with the same riddle order but different halves of riddles clued) were combined to form a misdirected-clued subject (with 10 clued riddles) and a misdirected-unclued subject (with 10 unclued riddles). In the ANOVAs, riddle order was used as a variable, but the effects and interactions with this variable were not reliable.

The results are easy to summarize. Subjects in the misdirected-clued condition did marginally less well than the informed group, showing some, but perhaps not full, benefit of the information. Subjects in the misdirectedunclued condition performed as well as the baseline group, showing no cost of the information. Finally, the misdirected group made good use of their clues, with performance .339 higher for the clued riddles than for the unclued riddles. (This last finding is important only for verifying that the clues were used.)

For the clued riddles, the proportions of congruent solutions were .831 and .758 for the informed and misdirected-clued groups, respectively $[F(1,48)=2.55$, $\left.M S_{e}=.027, p<.15\right]$. As can be seen in Figure 3, this difference held about equally at all the time intervals, but was never reliable [all $F \mathrm{~s}(1,48)$ between 1.58 and 2.94 ]. The mean solution-time difference $(2.34$ vs. 2.83$)$ just missed statistical significance $\left[F(1,48)=3.95, M S_{\mathrm{e}}=\right.$ $.81, p<.052$ ]. As can be seen in Table 3, the informed group performed slightly but consistently better than the misdirected-clued group (higher proportions on 8 of 9 riddles and faster on 8 of 10 ). One possibility is that this difference is related to the order of the riddles (i.e., perhaps the effect occurs for the first riddle only or for the last few riddles only). In a number of analyses, the effects of position were examined, but there was no consistent trend with riddle position.

For the unclued riddles, the proportions of congruent solutions were .419 and .357 for the misdirected-unclued and baseline groups, respectively $\left[F(1,48)=1.10, M S_{\mathrm{e}}=\right.$

Table 3

Percentage of Congruent Solutions and Mean Time Interval of Solutions in Experiment 3 for Each Riddle, Including Riddles Not Solved

\begin{tabular}{|c|c|c|c|c|c|c|c|c|}
\hline \multirow[b]{4}{*}{ Riddle } & \multicolumn{8}{|c|}{ Group } \\
\hline & & & \multicolumn{4}{|c|}{ Misdirected } & \multirow{2}{*}{\multicolumn{2}{|c|}{ Baseline }} \\
\hline & \multicolumn{2}{|c|}{ Informed } & \multicolumn{2}{|c|}{ Clued } & \multicolumn{2}{|c|}{ Unclued } & & \\
\hline & Percentage & Time & Percentage & Time & Percentage & Time & Percentage & Time \\
\hline 1 & 89 & 2.04 & 85 & 2.16 & 58 & 4.31 & 46 & 4.73 \\
\hline 2 & 77 & 3.43 & 65 & 3.89 & 31 & 5.54 & 31 & 5.65 \\
\hline 3 & 65 & 3.31 & 50 & 4.50 & 42 & 4.81 & 27 & 5.62 \\
\hline 4 & 46 & 4.65 & 46 & 4.43 & 54 & 4.23 & 42 & 4.92 \\
\hline 5 & 92 & 1.77 & 96 & 1.35 & 12 & 6.58 & 19 & 6.65 \\
\hline 6 & 92 & 1.54 & 85 & 1.96 & 15 & 6.35 & 15 & 6.47 \\
\hline 7 & 100 & 1.19 & 96 & 1.23 & 58 & 4.35 & 35 & 5.43 \\
\hline 8 & 100 & 1.00 & 96 & 1.31 & 31 & 5.50 & 50 & 4.38 \\
\hline 9 & 85 & 2.31 & 77 & 2.69 & 62 & 4.07 & 31 & 5.50 \\
\hline 10 & 85 & 2.19 & 62 & 4.04 & 58 & 4.39 & 62 & 4.31 \\
\hline
\end{tabular}

Note $-n=26$ /group; Riddles $4-10$ are same as in the other experiments. Riddles 1-3 were buffer riddles in the previous experiments. The actual orders of riddles were $(4,5,6,7,8,2,1,3,9,10)$ and $(5,4,7,6,1,9,8,10,2,3)$; Percentage = percent of congruent solutions; Time = mean solution-time interval, where Interval 1 is $0-20 \mathrm{sec}$, Interval 2 is $21-40 \mathrm{sec}$, up to Interval 6 for riddles solved in 101-120 sec. A score of 7 was assigned for riddles for which no congnuent solution was given. Because the hypothesis concerns overall time differences, it is appropriate to include nonsolutions. The time for just-solved riddles, as in the other tables, may be easily calculated, because the proportion of nonsolutions is 1 minus the proportion correct. 
$.045]$. Figure 3 shows that the difference was very small at the early intervals and grew only a little for the later intervals. None of these differences was reliable [all $F_{\text {s }}(1,48)<1.901$, except for the fourth interval, which was marginally reliable $[F(1,48)=2.79]$. The riddle data in Table 3 show that there was little difference in proportions ( 5 of 8 higher in misdirected-unclued). The mean solution intervals were very slightly faster in the misdirected-unclued condition (5.02 vs. 5.36 and faster on 7 of 10 riddles), but the differences were not reliable $\left[F(1,48)=1.52, M S_{e}=1.05\right]$. Riddle position again showed no differences.

In summary, this preliminary investigation of the retrieval process indicates some (though probably not full) benefit and no cost of the misdirected group being informed for only some of the riddles. Though the benefits (comparisons to informed subjects) were equivocal, the slight superiority of the misdirected-unclued subjects over the baseline subjects makes it unlikely that there was a cost for the unclued riddles.

Although the properties of memory retrieval in these tasks require further study, it is worthwhile to briefly consider the implications of these findings. The fact that being told that the earlier sentences might be relevant produces no cost for the unclued riddles is inconsistent with any view that claims that the search focuses first on these sentences or with any view that claims that the inclusion of the sentences might reduce the speed or effectiveness of the other search.

Analysis of the benefits constrains these explanations, however, for the misdirected-clued subjects did not perform quite as well as the informed subjects. Further research is needed to assess whether this marginal difference replicates, but we can briefly consider the implications of either finding. If the difference does not replicate and full benefit is found, the misdirected subjects get the best of both situations. Such a finding would suggest that neither means of problem solving interferes with the other. This result would be consistent with most models positing independent parallel searches, and might result in the pop-out phenomenology noted by Perfetto et al. (1983). If the difference between the informed and misdirected-clued groups occurs in later experiments as well, the picture would be more complicated. The fact that there is only some benefit would also be inconsistent with the simple view that the clue sentences are searched first. (It is not inconsistent with the idea that this search is first only some of the time, but then one would still expect some cost.) Although the no-cost result appears to call for a process that is not interfered with when the potentially relevant information is not useful, a result of only some benefit would mean that when the information is relevant, it is not retrieved exactly as in the informed condition. We suppose that a number of explanations that would meet this interaction could be devised (e.g., if the answer does not come immediately, informed subjects might have a higher probability of persisting because of their stronger belief in the relevance of the earlier sen- tences), but further research would be needed to determine the correct explanation. The important point is that finding some or full benefit in conjunction with no cost would place strong constraints on retrieval.

\section{GENERAL DISCUSSION}

In this section, we summarize the main findings and discuss the implications of two of the new findings. In Experiment 1, although Bowden's (1985) findings of informed-uninformed equivalence and changes with time were not replicated, differences between the uninformed and the baseline groups' performance were found. However, a simple mixture model and the protocols of some pilot subjects suggested that the uninformed subjects might have been catching on to the relevance of the sentences by noticing the similarity between their early answers and the sentences. The results of Experiment 2 showed that when the early riddles were moved to the end, the informed-uninformed difference remained, the uninformed-baseline difference was slightly reduced, and the mixture model again closely predicted performance in the uninformed condition. Thus, these two experiments provide further support for the difficulty of spontaneously noticing relevant information (without catching on) during problem solving in this paradigm, and cast doubt on the idea that the only difference between the performance of uninformed and informed subjects is time. This result is important because a belief in the time explanation would lead to very different research than a belief in a more qualitative difference between the uninformed and the informed subjects. Experiment 3 was a preliminary investigation of the retrieval properties in this paradigm. The group that had only some of their riddles clued performed marginally worse than the informed group on the clued riddles, but equivalently to the baseline group on the unclued riddles. Thus, there was some (and perhaps, full) benefit of the clue, but no cost when the earlier sentences were not appropriate.

The finding that cluing resulted in no cost places some constraints on retrieval explanations. As mentioned earlier, the data are inconsistent with simple search models in which the potentially relevant information is searched first. However, the findings are still consistent with a host of other models in which the knowledge of relevance does not interfere with solutions from default problem-solving activities. Further constraints can come from the benefit analysis (informed vs. misdirected-clued), but unfortunately, the results were equivocal. Although further research is needed to establish the exact pattern of cost and benefit, we hope that this preliminary experiment has made clear the usefulness of such research.

The other new finding (which is also mentioned for different purposes in Lockhart et al., 1988) is that subjects may have caught on to the relevance of the earlier sentences they rated by noticing the similarity between an answer to one of the riddles and one of these sentences. In these experimental studies, one might consider such 
an occurrence annoying, since it interferes with the analysis of interest (e.g., such an occurrence would have no effect if we collected only one observation per subject). In addition, one might argue that the paradigm has a very unusual structure in that the subject sees two sets of information (sentences and riddles) that are unrelated within each set, but are related across sets. However, we argue here that this new finding has important implications for the issues under examination and for some of the situations that these experimental studies are meant to capture.

As reviewed earlier, many studies have shown that people fail to access relevant information when problem solving. Much of what we learn appears to be stored with respect to the context in which it is learned, and its access in a different context is difficult (e.g., Gick \& Holyoak, 1980, 1983; Lockhart et al., 1988; Ross, 1989b; Spencer \& Weisberg, 1986). What allows people to think back to previously learned relevant information? Over the last 10 years, a number of researchers have examined how problem solvers are reminded of earlier examples or other relevant information when solving problems. In the work on analogous problems, the consensus is that remindings are affected both by structural similarities between the problems (i.e., those aspects that are relevant to the problems' solutions) and by superficial similarities between the problems (e.g., Holyoak \& Koh, 1987; Ross, 1987). In a similar vein, the recent work on accessing nonanalogous information has shown that access will increase if subjects can be cued to encode (and focus on) the relevant aspects (Stein et al., 1986) or if subjects process the information in a way that is similar to the way they will need to process it at the time the riddle is presented (e.g., Adams et al., 1988; Lockhart et al., 1988). Gentner and Landers (1985) and Stein et al. (1986) also showed that superficial similarities can affect access. For present purposes, the important point about this work on accessing analogous or other relevant information is that it has focused on situations in which the information accessed is a particular episode or instance (such as an earlier problem or a particular sentence read earlier).

In the present experiments, it appears that subjects may have caught on to the relevance of the earlier clues by noticing the similarity between an answer and the earlier sentence (presumably by some combination of structural and superficial similarity). Although this finding in some sense is specific to the paradigm, it brings up two interesting aspects of reminding that have not received much attention. First, remindings may serve not only to suggest a particular earlier episode as relevant, but also to suggest that the knowledge within the reminded domain may be relevant. That is, even if the exact information that one is reminded of does not prove useful, sometimes other knowledge from the reminded domain may be useful. Second, although noticing the similarity of a correct answer to other relevant information is not very helpful for solving the problem (because the answer provides the solution already), it may prove helpful in later problem solving. If there are other problems, or other times of related problem solving, the earlier postsolution noticing of relevance of that other information could be quite helpful.

An example may help to make these points clearer. Imagine that one late spring, a few months after buying your first home (so that your knowledge of house repair is not great), you went in the basement and found some wetness where the walls meet the floor. You might think about drainage problems associated with spring rains or possible plumbing problems. Now consider two scenarios, related to the two extended implications mentioned in the previous paragraph. First, suppose, perhaps after researching drainage and plumbing possibilities, you noticed that your glasses got misty if you stayed in the basement for a while. This fact might make you think about other times when your glasses have gotten misty, evoking some knowledge about condensation. If you were alert, you might realize that this knowledge about condensation could also be relevant to the basement wetness. Once you realized that this knowledge might be relevant, you could bring to bear your knowledge about heat and dampness to suggest the possibility that a dehumidifier might solve the wetness problem. Although the reminding did not lead to the answer immediately, it suggested the relevance of some domain of knowledge which could be used to arrive at a solution.

For the second scenario, suppose that some more experienced homeowner suggested this possibility to you, and you found that the dehumidifier did solve the problem. (Or, to make it closer to the present finding, suppose you saw a dehumidifier solving the problem in someone else's house and realized why and what knowledge was relevant.) If you have a wetness problem elsewhere in the house, it seems likely that you would consider whether the knowledge relevant to the basement problem would be relevant to this other problem. In addition, if you had a wetness problem in the future, it seems likely that knowledge about water condensation would be more likely to be considered than if you had not noticed its relevance to this basement experience. ${ }^{10}$

Returning to the present studies, the answers to these riddles were quite similar (in both meaning and wording) to the sentences rated earlier, and having solved one riddle might well remind one of an earlier sentence. Although the subjects' catching on to the relevance of the sentences contaminated the intended experimental manipulation, it has led us to consider that (1) remindings that do not lead immediately to a solution may still serve to suggest the relevance of domains, not just instances, for the solution and (2) postsolution remindings may affect subsequent problem solving. These possibilities will require further investigation, but they appear to be plausible and littlestudied uses of remindings. The noticing of similarity between some knowledge and some problem-solving product 
may be one means by which we are able to access relevant information in our everyday problem solving.

\section{REFERENCES}

Adams, L. T., Kasserman, J. E., Yearwood. A. A., Perfetto, G. A., Bransford, J. D., \& Fanks, J J. (1988). Memory access: The effects of fact-oriented versus problem-oriented acquisition. Memory \& Cognition, 16, 167-175.

Anderson, J. R., \& Thompson, R. (1989). Use of analogy in a production system architecture. In S. Vosniadou \& A. Ortony (Eds.), Similarity and analogical reasoning (pp. 267-297). Cambridge, England: Cambridge University Press.

BOWDEN, E. M. (1985). Accessing relevant information during problem solving: Time constraints on search in the problem space. Memory \& Cognition, 13, 280-286.

DELla ROSA, D. (1985). Abstraction of problem-type schemata through problem comparison (Rep. No. 146). Boulder, CO: University of Colorado, Institute of Cognitive Science.

Gardner, M. (1978). Aha! Insight. New York: Freeman

GenTNer, D., LANDERS, R. (1985, November). Analogical reminding: A good match is hard to find. Paper prepared for the panel on Commonsense Reasoning at the International Conference on Systems, Man \& Cybernetics, Tucson, AZ

Gick, M. L., \& Holyoak, K. J. (1980). Analogical problem solving. Cognitive Psychology, 12, 306-355.

GiCK, M. L., \& HolyoAk, K. J. (1983). Schema induction and analogical transfer. Cognitive Psychology, 14, I-38.

Holyonk, K. J., \& KoH, K. (1987). Surface and structural similarity in analogical transfer. Memory \& Cognition, 15, 332-340.

Holyoak, K. J., \& ThagaRd, P. (1989). A computational model of analogical problem solving. In S. Vosniadou \& A. Ortony (Eds.) Similarity and analogical reasoning (pp. 242-266). Cambridge, England: Cambridge University Press.

Lockhart, R. S., LAmon, M., Gick, M. L. (1988), Conceptual transfer in simple insight problems. Memory \& Cognition, 16, 36-44.

Perfetto, G. A., Bransford, J. D., \& Franks, J. J. (1983). Constraints on access in a problem solving context. Memory \& Cognition, 11, 24-31.

ReEd, S. K., ERnest, G. W., \& Baneri, R. (1974). The role of analogy in transfer between similar problem states. Cognitive Psychology, 6, 436-450.

RoSENBLOOM, J. (1976). Biggest riddle book in the world. New York: Sterling.

Ross, B. H. (1984). Remindings and their effects in learning a cognitive skill. Cognitive Psychology, 16, 371-416.

Ross. B. H. (1987). This is like that: The use of earlier problems and the separation of similarity effects. Journal of Experimental $P_{s y c h o l}$ ogy: Learning, Memory, \& Cognition, 13, 629-639.

Ross, B. H. (1989a). Distinugishing types of superficial similarities: Different effects on the access and use of earlier problems. Joumal of Experimental Psychology: Learning. Memory, \& Cognition, 15. 456-468.

Ross, B. H. (1989b). Remindings in learning and instruction. In S. Vosniadou \& A. Ortony (Eds.), Similarity and analogical reasoning (pp. 438-469). Cambridge, England: Cambridge University Press.

Ross, B. H., \& KeNNEDY, P. T. (in press). Generalizing from the use of earlier examples in problem solving. Journal of Experimental Psychology: Learning, Memory, \& Cognition.

Ross, B. H., Perkins, S. J. \& Tenpenny, P. L. (1989). Remindingbased category learning. Manuscript submitted for publication.

Spencer, R. M., \& Weisberg, R. W. ([986). Context-dependent effects on analogical transfer. Memory \& Cognition, 14, 442-449.

Stein, B. S., Way, K. R., Benningliel., S. E., \& Hedgecolitih. C. A. (1986). Constraints on spontaneous transfer in problem-solving tasks. Memory \& Cognition, 14, 432-441

Wesiberg, R. W., \& Alba, J. W. (1981). An examination of the al leged role of "fixation" in the solution of several "insight" problems. Journal of Experimental Psychology: General, 110, 169-192.

Wesiberg, R., DiCamil.1o, M. \& Phill.tPs, D. (1978). Transferring old associations to new situations: A nonautomatic process. Journal of Verbal Learning \& Verbal Behavior, 17, 219-228.

\section{NOTES}

1. The use of one order of riddles always leaves open the possibility that any results may be due to the particular order used. However. because the results of Bowden's (1985) study cast doubt on a large number of earlier findings, we thought it important to replicate his study closely.

2. Both Experiments 1 and 2 started out as studies using half the number of subjects (i.e., about 20 subjects/condition), but were extended when it became clear that Bowden's (1985) results were not being well replicated. The results have been combined for ease of exposition and to gain more power. The data were quite similar across each half, with the one exception noted in Note 8, below

3. Paired-comparison analyses were conducted using $t$ tests as in Bowden's (1985) study to ensure that any difficulties in the replications were not due to differences in statistics.

4. The uninformed group's advantage over baseline for the early intervals is inconsistent with Perfetto et al.'s (1983) findings as well. However, the riddles were not exactly the same, and the clues in the present experiment appear to have had more overlap with the riddles and to have been more blatant than in the earlier study.

5 . Because of the large differences in proportions among the groups, we decided to exclude nonsolutions from the tables. Since the proportions and number of observations for each riddle are known, the reader may easily calculate the mean interval including nonsolutions.

6. This modified mixture model could not be applied to Bowden's (1985) data because there is no information about the riddle at which the subjects thought they caught on. The modified version of the model would lead to a worse fit for the overall proportion since the prediction is underestimating already. However, the change in fit would be small. For example, assuming the subjects caught on after the first riddle, the prediction would be .500 for the observed .54 , instead of .514 . The overall interval, though, would be slightly closer, with a prediction of 4.37 for the observed 4.40 , instead of the unmodified prediction of 4.26 .

7. To ensure that any change from Experiment 1 was due to the moving of the first three riddles, we again chose not to randomize the riddle order.

8. The two subexperiments showed slightly different results for the informed-uninformed difference, with one having a reliable effect and the other a small difference that did not approach statistical significance. Otherwise, the data were quite similar and much of the discrepancy could be traced to differences in when the subjects in the uninformed condition claimed to have caught on. The modified mixture model fit each part well, though not as well as it fit the combined data.

9. We thank Robert Weisberg for this suggestion.

10. Depending upon how it occurs, this postsolution noticing of relevant information may affect subsequent problem solving either by increasing the accessibility of the earlier episode (i.e., increasing the probability of being reminded of that episode) or by leading to the formation of some generalized knowledge about this type of situation. This distinction is not discussed further here because it is not directly germane, but remindings have been shown to lead to generalizations in problem solving (Ross \& Kennedy, in press) and category learning (Ross, Perkins, $\&$ Tenpenny, 1989).

\section{APPENDIX A}

\section{The Modified Mixture Model and Fits to Experiments 1 and 2}

The fitting of the modified mixture model is simple. Using the subjects' reports. we count the number of riddles for which they are presumed to be like baseline subjects (all the ones up io and including the riddle at which they claim to have caught on) and the ones for which they are presumed to be like informed subjects (all other riddles). Thus, if a subject claims to have caught on at the first riddle. we consider the data to be 1 base- 
line riddle and 9 informed riddles. After all the riddles are counted, we take the proportion of baseline riddles and multiply by the baseline group's performance, and take the proportion of informed riddles and multiply by the informed group's performance. The predicted uninformed score is this weighted average (i.e., the sum of these two products).

To evaluate the fit of the model we compute $\chi^{2}$ using the observed and expected frequencies from each of the seven cells (the riddles solved during the six time intervals plus the unsolved riddles). Thus there are six degrees of freedom. It should be noted that although the proportions given in the tables that follow are cumulative, the frequencies used to compute $\chi^{2}$ were not cumulative. For example, the observed frequency for Interval 4 would include only those riddles solved during Interval 4 rather than the riddles solved during Intervals 1 through 4. As an additional index of fit, we also compute the correlation between the observed and predicted frequencies and determine the percentage of variance accounted for. In evaluating the fit, it should be noted that since each subject contributes 10 observations (one for each riddle), the observations are not strictly independent.

\section{Experiment 1}

In this experiment, 29 of the 40 subjects claimed to have caught on: 15 claimed to have caught on at the first riddle, 8 on the second, 1 on the third, 2 on the sixth, 1 on the seventh, 1 on the eighth, and 1 on a buffer riddle (this final subject was assumed to be totally informed). Thus, 171 of the 400 riddles (.4275) were "baseline" and the rest (.5725) were "informed."

The observed and predicted values for the time intervals were:

\begin{tabular}{lrrrrrr} 
& 1 & 2 & 3 & 4 & 5 & \multicolumn{1}{c}{6} \\
\cline { 2 - 7 } Baseline & .280 & .378 & .440 & .470 & .495 & .518 \\
Informed & .585 & .703 & .743 & .773 & .788 & .798 \\
Uninformed & & & & & & \\
$\quad$ Observed & .428 & .548 & .593 & .625 & .630 & .645 \\
$\quad$ Predicted & .455 & .564 & .613 & .643 & .662 & .678 \\
Difference & -.027 & -.016 & -.020 & -.018 & -.032 & -.033
\end{tabular}

Although there appears to be a consistent overestimate of performance, the differences are not large and the fit is quite good $\left[x^{2}(6)=6.83, p>.3 ; r^{2}=.988\right]$.

\section{Experiment 2}

In this experiment, 30 of the 41 subjects claimed to have caught on. By Riddle Position 1 to 10 , the number of subjects catching on at each riddle was $2,3,3,5,2,3,0,4,3$, and 3 . (Remember, the riddles that were in Positions 1, 2, and 3 in Experiment 1 are now in Positions 8, 9, and 10.) In addition, 2 subjects claimed to have caught on at a buffer riddle and were considered to be fully informed. Thus, 264 of the 410 riddles (.644) were "baseline" and the rest (.356) were "informed."

The predicted and observed values for the time intervals were:

\begin{tabular}{lcccccc} 
& 1 & 2 & 3 & 4 & 5 & 6 \\
\cline { 2 - 7 } Baseline & .278 & .383 & .424 & .446 & .459 & .481 \\
Informed & .510 & .663 & .693 & .712 & .729 & .734 \\
Uninformed & & & & & & \\
$\quad$ Observed & .383 & .485 & .527 & .554 & .563 & .578 \\
Predicted & .361 & .483 & .520 & .541 & .555 & .571 \\
Difference & .022 & .002 & .007 & .013 & .008 & .007
\end{tabular}

The predictions are all very close to the observed proportions, although there is a slight tendency toward underpredicting in each case. Again, the differences are small and the fit is excellent $\left[\chi^{2}(6)=3.34, p>.7 ; r^{2}=.995\right]$.

\section{APPENDIX B}

\section{Analysis by Riddle Halves for Experiments 1 and 2}

Bowden (1985) argued that if uninformed subjects caught on, "one would expect the change in their first-half to second-half solution times to be more positive (or less negative) than the changes of the informed and baseline subjects" (p. 284). He found no evidence for this interaction. The means indicated that the uninformed group showed the most negative change (the first half contained easier riddles). In this Appendix, we provide the corresponding means for our Experiments 1 and 2. As can be seen from the means, no formal analysis is necessary.

\section{Experiment 1}

We calculated the mean solution intervals (with a 7 assigned for nonsolutions) for the first five and the second five riddles for each group:

\begin{tabular}{lccc}
\multicolumn{1}{c}{ Group } & First Five & Second Five & Difference \\
Baseline & 4.07 & 4.78 & -.71 \\
Uninformed & 3.10 & 3.97 & -.87 \\
Informed & 2.47 & 2.75 & -.28
\end{tabular}

Clearly, although the differences between groups are small, the uninformed group again shows the most negative change, not the least negative.

\section{Experiment 2}

Now, the easy riddles tend to be in the second half, but the calculations are made in the same way:

\begin{tabular}{lccc}
\multicolumn{1}{c}{ Group } & First Five & Second Five & Difference \\
\hline Baseline & 5.29 & 3.76 & 1.53 \\
Uninformed & 5.03 & 2.78 & 2.25 \\
Informed & 4.12 & 1.79 & 2.33
\end{tabular}

Although the uninformed group may improve more than the baseline group, it clearly does not improve more than the informed group.

In summary, considered along with the evidence suggesting that a large portion of uninformed subjects are catching on, this analysis does not appear to be sensitive to catching on. For the results presented in Bowden's (1985) study and the first study in the present paper, this lack of sensitivity may be because subjects caught on so quickly. The reason for its insensitivity in the second study is not clear; however, there is the indication of a difference from the baseline subjects.

\section{APPENDIX C}

\section{Additional Riddles and Clues Used in Experiment 3}

1. A police officer had a brother, but the brother had no brother. How is that possible?

Clue: If a woman has a brother, then that man has a sister.

2. A man says he has two U.S. coins in his hand which total fifty-five cents. One is not a nickel. What are the two coins? 
Clue: If there are two coins and one is not a dime, the other one is.

3. Two fathers and two sons went duck hunting. Each shot a different duck, but they shot only three ducks in all. How is that possible?

Clue: A father has a father.
Note-These riddles were used as buffers in Experiments 1 and 2 , but were exchanged with Bowden's first three riddles for the final study.

(Manuscript received September 1, 1988; revision accepted for publication January $18,1989$.

\title{
Announcement
}

\author{
30th Annual Meeting of the Psychonomic Society \\ Atlanta, Georgia \\ November 17-19, 1989
}

The 30th Annual Meeting of the Psychonomic Society will be held in Atlanta, Georgia, November 17 19, 1989. The meetings will begin Friday morning and continue until Sunday at noon. The headquarters hotel will be the Hyatt Regency.

The program and hotel reservation cards will be mailed to members and associates in September. A copy of the program will be published in the November issue of the Bulletin of the Psychonomic Society.

For further information, please contact the secretary-treasurer of the Society: Michael E. Rashotte, Department of Psychology, Florida State University, Tallahassee, FL 32306-1051 (Telephone: 904-644-2040; BITNET: Rashot@FSU). 